Psychother Psychosom 2018;87:243-245

DOI: $10.1159 / 000489044$

\title{
Individual Negative Affective Trajectories Can Be Detected during Different Depressive Relapse Prevention Strategies
}

\author{
Christien Slofstra ${ }^{\mathrm{a}}$ Maaike H. Nauta ${ }^{\mathrm{a}}$ Laura F. Bringmann ${ }^{\mathrm{b}, \mathrm{c}}$ \\ Nicola S. Klein ${ }^{\text {a }}$ Casper J. Albers ${ }^{\mathrm{b}}$ Nikolaos Batalas ${ }^{\mathrm{d}}$ \\ Marieke Wichers ${ }^{c}$ Claudi L.H.Bockting ${ }^{\mathrm{a}, \mathrm{e}}$ \\ ${ }^{a}$ Department of Clinical Psychology and Experimental \\ Psychopathology, University of Groningen, Groningen, \\ The Netherlands; ${ }^{b}$ Department of Psychometrics and Statistics, \\ University of Groningen, Groningen, The Netherlands; \\ 'Interdisciplinary Center Psychopathology and Emotion \\ Regulation (ICPE), Department of Psychiatry (UCP), University \\ Medical Center Groningen (UMCG), University of Groningen, \\ Groningen, The Netherlands; ${ }^{d}$ Department of Industrial \\ Design, Eindhoven University of Technology, Eindhoven, \\ The Netherlands; ${ }^{e}$ Department of Psychiatry, Academic Medical \\ Centre, University of Amsterdam, Amsterdam, The Netherlands
}

Meta-analyses demonstrate that different relapse prevention strategies, including continuation of antidepressant medication [1], preventive psychological therapy [2], or the combination of both [3] reduce the risk of relapse at a group level. However, the average effect of a treatment does not apply to every individual in that group [4], and many individuals experience a subsequent episode despite their use of relapse prevention strategies [3]. One of the current challenges is to personalize relapse prevention strategies [5].

For advancing personalized relapse prevention strategies, zooming into within-individual affective trajectories may be the way forward [6]. Within-individual affective changes may characterize transitions into and out of a depressive state [7]. It is hypothesized that these transitions differ from person to person, that gradual transitions into a depressive state are characterized by increases in mean negative affect, and that abrupt transitions into a depressive state are preceded by increased negative affective inertia [8]. Negative affective inertia refers to the degree one's affect is predictive of itself over time and thus indicates that current levels of negative affect predict negative affect levels at the next time point [9].

The empirical support for these hypotheses is limited though promising and suggests that increased affective inertia may indeed signal an abrupt transition into a depressive state [7]. However, to our knowledge, no studies to date examined whether significant within-individual affective change could be detected in a group of previously depressed individuals undergoing different relapse prevention strategies.

\section{KARGER}

E-Mail karger@karger.com www.karger.com/pps (c) 2018 The Author(s)

Published by S. Karger AG, Basel
Karger

Open access

This article is licensed under the Creative Commons AttributionNonCommercial-NoDerivatives 4.0 International License (CC BY NC-ND) (http://www.karger.com/Services/OpenAccessLicense). Usage and distribution for commercial purposes as well as any distribution of modified material requires written permi
The primary aim of this study (see Slofstra et al. [6] for details on the design) is to explore, using experience sampling methodology (ESM), whether individual negative affective trajectories can be detected in remitted previously depressed individuals undergoing different relapse prevention treatments. It was hypothesized that affective trajectories would vary from person to person. A second aim was to tentatively explore whether these individual trajectories during treatment may be relevant for subsequent depressive relapse. It was hypothesized that increases in mean negative affect or negative affective inertia would be discerned in a subset of individuals that relapsed. Furthermore, decreases in mean negative affect or negative affective inertia were hypothesized to signal decreased vulnerability for depressive relapse.

Affect was repeatedly assessed in daily life for 8 weeks, using ESM. Sufficient assessments to be eligible for analyses were provided by 42 (out of 72) previously depressed participants, and 11 (out of 15) matched never depressed controls. Previously depressed individuals had been randomly assigned to continuation of antidepressants $(n=10)$, continuation of antidepressants with preventive cognitive therapy $(n=15)$, or tapering of antidepressants with preventive cognitive therapy $(n=17)$.

To explore whether individual trajectories in negative affect can be detected, the presence of significant change in mean negative affect or negative affective inertia was analysed per individual [10]. Additional information about the methods and results can be found in the supplemental materials (for all online suppl. material, see www.karger.com/doi/10.1159/000489044). Table 1 summarizes the changes in mean negative affect or negative affective inertia per group. These results show that individual trajectories while receiving various relapse prevention treatments can be detected and that these affective changes indeed vary from person to person. As a striking example, 2 individuals in 1 group experienced increases in mean negative affect while 2 others experienced decreases. Finally, 1 never depressed individual participant from the matched control group demonstrated decreased negative affective inertia.

For the secondary aim of this study, it was descriptively explored whether individual affective trajectories are related to depressive relapse as assessed using repeated clinical interviews over 15 months. The expected increases in mean negative affect were observed in 2 (out of $42=5 \%$ ) previously depressed individuals, both of whom relapsed. However, no increases in negative affective inertia were observed in any previously depressed individuals, including those who relapsed at the end or soon after the ESM study period. Of the 42 previously depressed participants, 22 relapsed (52\%). Thus, increases in mean negative affect were observed in a small minority of previously depressed individuals that relapsed (2 out of $22=9 \%$ ). Nine previously depressed individuals displayed decreases in mean negative affect or negative affective inertia (21\%). Of these 9 individuals, 5 (56\%) did subsequently relapse. Unexpectedly, a decrease in negative affective inertia co-occurred

Claudi L.H. Bockting

Department of Psychiatry, Academic Medical Centre, University of Amsterdam Meibergdreef 5

NL-1105 AZ Amsterdam (The Netherlands)

E-Mail c.l.bockting@ amc.uva.nl 
Table 1. Summaries of within-individual negative affective trajectories

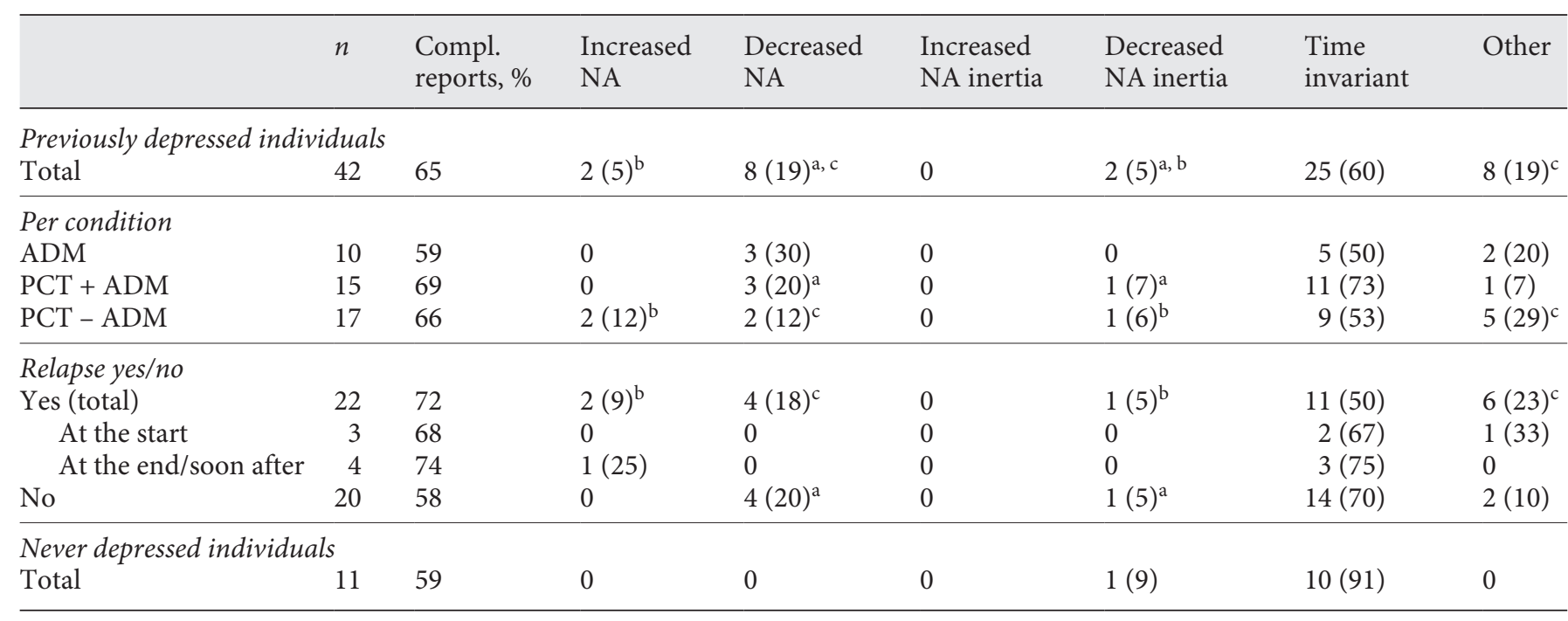

Figures in parentheses are percentages. Compl. reports, percentage of completed reports; NA, negative affect; Time invariant, the time series did not demonstrate a structural change of time (fluctuations over time may have been present); other, model could not be fitted or interpreted; ADM, assigned to the antidepressant medication continuation arm of the trial; PCT + ADM, assigned to the antidepressant medication continuation in combination with preventive cognitive therapy arm of the trial; PCT - ADM, assigned to the arm of the trial that combines preventive cognitive therapy with tapering of antidepressant medication; at the start, relapse during the first 4 weeks of the ESM study period; soon after, relapse at the end of or within 3 weeks after the ESM study period. ${ }^{a}$ One individual (patient 14) displayed both decreased negative affect and negative affective inertia. ${ }^{\mathrm{b}}$ One individual (patient 26) experienced increased negative affect and decreased negative affective inertia. ${ }^{c}$ One individual (patient 39 ) demonstrated decreased negative affect while the analyses could not be interpreted in terms of invariant or changing inertia. Because of particular patients (superscripts a $+b+c)$, not all rows add up to $100 \%$. Details on analyses per individual can be found in the supplemental materials.

with an increase in mean negative affect in 1 previously depressed individual.

Thus, although individual negative affective trajectories can be observed in previously depressed individuals undergoing different relapse prevention strategies, the results cast doubt upon their presumed relevance for depressive relapse in recurrent depression. A limitation of the current study is that the ESM study period was limited to 8 weeks while many individuals relapsed many months later. Continuing the high-intensive ESM procedure for years might be too burdensome and unpractical and therefore unfeasible. However, to examine the clinical relevance of affect dynamics and individual trajectories in depression, individual affective trajectories need to be monitored over a longer period of time in larger patient samples. If individual trajectories indeed precede relapse, it remains to be investigated whether individual affective trajectories can be an early warning signal that offers the potential to prevent a subsequent depressive episode.

In summary, results in this relatively large sample of remitted recurrently depressed individuals demonstrate that individual affective trajectories while receiving relapse prevention treatments vary from person to person and can be assessed using ESM. On the one hand, this might open up the possibility of tailoring interventions to individual affective trajectories. On the other hand, within-individual increases in mean negative affect were only found in a very small proportion (9\%) of previously depressed individuals who subsequently relapsed. Moreover, most (56\%) individuals who demonstrated decreases in mean negative affect or negative affective inertia nevertheless relapsed. These results therefore call for future research to investigate whether these individual trajectories are clinically meaningful.

\section{References}

1 Glue P, Donovan MR, Kolluri S, Emir B: Meta-analysis of relapse prevention antidepressant trials in depressive disorders. Aust NZ J Psychiatry 2010;44:697-705.

-2 Biesheuvel-Leliefeld KE, Kok GD, Bockting CLH, Cuijpers P, Hollon SD, van Marwijk HW, et al: Effectiveness of psychological interventions in preventing recurrence of depressive disorder: meta-analysis and metaregression. J Affect Disord 2015;174:400-410.

3 Guidi J, Tomba E, Fava GA: The sequential integration of pharmacotherapy and psychotherapy in the treatment of major depressive disorder: a meta-analysis of the sequential model and a critical review of the literature. Am J Psychiatry 2016;173:128-137.

4 Verhagen S, Hasmi L, Drukker M, van Os J, Delespaul P: Use of the experience sampling method in the context of clinical trials. Evid Based Ment Health 2016;19:86-89.

-5 Kazdin AE: Evidence-based psychosocial treatment: advances, surprises, and needed shifts in foci. Cogn Behav Pract 2016;23:426-430. 
-6 Slofstra C, Klein NS, Nauta MH, Wichers M, Batalas N, Bockting CLH: Imagine your mood: study design and protocol of a randomized controlled micro-trial using app-based experience sampling methodology to explore processes of change during relapse prevention interventions for recurrent depression. Contemp Clin Trials Commun 2017;7:172-178.

7 Wichers M, Groot PC; Psychosystems, ESM Group, EWS Group: Critical slowing down as a personalized early warning signal for depression. Psychother Psychosom 2016;85:114-116.
8 Van de Leemput IA, Wichers M, Cramer AO, Borsboom D, Tuerlinckx F, Kuppens P, et al: Critical slowing down as early warning for the onset and termination of depression. Proc Natl Acad Sci USA 2014;111:87-92.

-9 Kuppens P, Allen NB, Sheeber LB: Emotional inertia and psychological maladjustment. Psychol Sci 2010;21:984-991.

10 Bringmann LF, Hamaker EL, Vigo DE, Aubert A, Borsboom D, Tuerlinckx F: Changing dynamics: time-varying autoregressive models using generalized additive modeling. Psychol Methods 2017;22:1-17. 